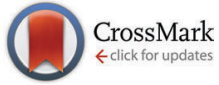

Cite this: Phys. Chem. Chem. Phys., 2014, 16, 27037

Received 22nd September 2014, Accepted 28th October 2014

DOI: $10.1039 / c 4 c p 04260 c$

www.rsc.org/pccp

\title{
A homodimeric BODIPY rotor as a fluorescent viscosity sensor for membrane-mimicking and cellular environments
}

\author{
Sangram Raut, ${ }^{a}$ Joseph Kimball, ${ }^{a}$ Rafal Fudala, ${ }^{\mathrm{b}}$ Hung Doan, ${ }^{\mathrm{a}}$ Badri Maliwal, ${ }^{\mathrm{b}}$ \\ Nirupama Sabnis, ${ }^{c}$ Andras Lacko, ${ }^{c}$ Ignacy Gryczynski, ${ }^{b}$ Sergei V. Dzyuba ${ }^{d}$ and \\ Zygmunt Gryczynski*ab
}

\begin{abstract}
Fluorescence properties of a novel homodimeric BODIPY dye rotor for Fluorescence Lifetime Imaging Microscopy (FLIM) are reported. Steady state and time resolved fluorescence measurements established the viscosity dependent behaviour in vitro. Homodimeric BODIPY embedded in different membrane mimicking lipid vesicles (DPPC, POPC and POPC plus cholesterol) is demonstrated to be a viable sensor for fluorescence lifetime based viscosity measurements. Moreover, SKOV3 cells readily endocytosed the dye, which accumulated in membranous structures inside the cytoplasm thereby allowing viscosity mapping of internal cell components.
\end{abstract}

\section{Introduction}

A variety of mass and signal transport phenomena as well as intermolecular interactions are often governed by viscosity. ${ }^{1,2}$ As such, it is important to be able to measure/estimate viscosity and detect the changes in viscosity upon exposure to a stimulus. In view of synthetic accessibility, molecular viscometers are attractive probes for sensing the viscosity of various environments. ${ }^{3-5}$

Fluorophore dyads/dimers, which are small molecular probes with two fluorescent moieties, are an interesting class of molecular viscometers, which have found numerous applications in ratiometric sensing of analytes, cascade-type energy transfer events and chemical transformations. ${ }^{6-9}$ The spectroscopic properties of these types of systems, including extinction coefficients, apparent brightness and Stokes shifts, could be tuned via structural and functional modification of the monomeric fluorophores, and further accentuated by the dyad's structure. Since many biochemical applications are hindered by the lack of suitable structurally diverse fluorophores, the access to dyads, which rely on facile and modular synthetic approaches allowing for the formation of both homodimeric and heterodimeric systems, might provide a viable solution.

\footnotetext{
${ }^{a}$ Department of Physics and Astronomy, Texas Christian University, Fort Worth, TX, 76129, USA. E-mail: z.gryczynski@tcu.edu

${ }^{b}$ Department of Cell Biology and Immunology, UNT Health Science Center, Fort Worth, TX, 76107, USA

${ }^{c}$ Department of Integrative Physiology and Anatomy, UNT Health Science Center, Fort Worth, TX, 76107, USA

${ }^{d}$ Department of Chemistry, Texas Christian University, Fort Worth, TX, 76129, USA
}

BODIPY dyes are versatile fluorophores whose spectral properties can be tuned via a range of structural modifications. ${ }^{10,11}$ As a result, BODIPY dyes have been explored in a variety of applications, including molecular, ionic and biological sensing. ${ }^{12-15}$ Importantly, BODIPY-based rotors have been shown to be suitable for sensing viscosity, including the viscosity of intracellular environments. Specifically, BODIPY dyes with the modification in the para-position of the phenyl substituent and in the mesoposition of the BODIPY scaffold were shown to be viable sensors of intracellular viscosity as their fluorescent lifetime showed a good correlation with viscosity. ${ }^{16-19}$ It should be noted that the so-called "distorted-BODIPY" fluorescent viscometer with the carboxyaldehyde-moiety in the meso-position of the BODIPYscaffold was shown not only to map the viscosity of the cell, but also to detect viscosity changes associated with the early stages of apoptosis in a breast cancer cell line MCF-7. ${ }^{20}$ With regard to the BODIPY-based dyads for measuring intracellular viscosity, the coumarin-BODIPY dyad was demonstrated to detect viscosity in mitochondria. ${ }^{21}$ In view of the significant linear correlation of both fluorescence intensity and fluorescence lifetimes with viscosity, the aforementioned sensor was shown to be applicable for monitoring viscosity changes that occurred during mitochondrial apoptosis events. Overall, it could be argued that fairly long fluorescence lifetimes (on the order of several ns) along with synthetic accessibility make BODIPY-containing systems a viable platform for the development of fluorescence lifetime based molecular viscometers.

It should be pointed out that multistep synthetic protocols that are employed for the preparation of the dyads (BODIPYbased $^{21}$ as well as porphyrin-based, which have proven to be 


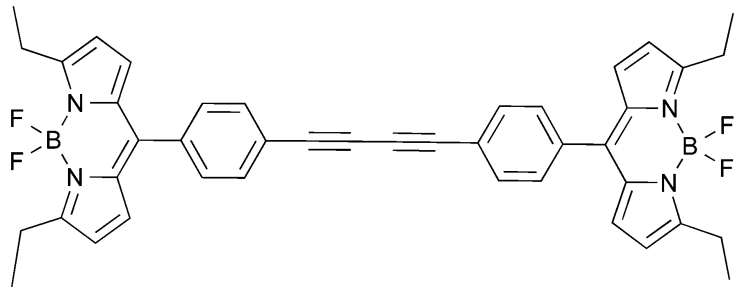

Fig. 1 Chemical structure of the BODIPY homodimer.

very potent in mapping cellular viscosity ${ }^{22}$ ) should be taken into consideration and they often might be viewed as a drawback. In order to overcome this drawback, we decided to explore homodimeric BODIPY dyes, which could be prepared in a few steps from readily available starting materials. Towards this end, we recently assessed the potential of a BODIPY dimer to act as a viscometer for molecular and ionic solvents (Fig. 1). ${ }^{23}$ Here, we expand on the application of the BODIPY dimer and report on its ability to act as a microviscosity sensor in various cellular and membrane-mimicking environments.

\section{Materials and methods}

\section{BODIPY dyad synthesis}

All chemicals and solvents were obtained from commercial sources; they were of highest grade possible and were used as received. The BODIPY homodimer was prepared according to the literature procedure, ${ }^{23}$ and exhibited spectral properties consistent with the structure: ${ }^{1} \mathrm{H}$ NMR $\left(400 \mathrm{MHz}, \mathrm{CDCl}_{3}\right): \delta=7.68(\mathrm{~d}, J=$ $8.4 \mathrm{~Hz}, 2 \mathrm{H}), 7.53(\mathrm{~d}, J=8.4 \mathrm{~Hz}, 2 \mathrm{H}), 6.75(\mathrm{~d}, J=4.2 \mathrm{~Hz}, 2 \mathrm{H}), 6.40$ (d, $J=4.3 \mathrm{~Hz}, 2 \mathrm{H}), 3.11$ (q, $J=7.6 \mathrm{~Hz}, 4 \mathrm{H}), 1.38$ (t, $J=7.6 \mathrm{~Hz}, 6 \mathrm{H})$; ${ }^{19} \mathrm{~F}$ NMR $\left(376 \mathrm{MHz}, \mathrm{CDCl}_{3}\right): \delta=145.24(\mathrm{q}, J=32.7 \mathrm{~Hz})$.

\section{Spectroscopic measurements}

UV-Vis absorption and fluorescence spectra were obtained using a Cary 50 bio UV-visible Spectrophotometer (Varian Inc.) and a Cary Eclipse Spectrofluorometer (Varian Inc.) respectively. All the measurements were done using $0.4 \mathrm{~mm}$ $\times 1 \mathrm{~cm}$ quartz cuvettes at room temperature with optical density below 0.05 , unless otherwise mentioned. In order to measure the quantum yield, absorption spectra of the BODIPY dimer was collected followed by measuring the integrated fluorescence intensity of the sample. A solution of fluorescein in 0.1 $\mathrm{M} \mathrm{NaOH}$ was used as a reference (quantum yield: 0.90 ). ${ }^{24}$ The fluorescence lifetime was measured on a FluoTime 200 fluorometer (PicoQuant, Inc.) using a $470 \mathrm{~nm}$ diode laser. The fluorometer was equipped with an ultrafast microchannel plate detector (MCP) from Hamamatsu, Inc. The fluorescence lifetimes were measured in the magic angle condition and data were analyzed using the FluoFit4 program from PicoQuant, Inc. (Germany) using the multi-exponential fitting model:

$$
I(t)=\sum_{i} \alpha_{i} \mathrm{e}^{-t / \tau_{i}}
$$

where $\alpha_{i}$ is the amplitude of the decay of the $i$ th component at time $t$ and $\tau_{i}$ is the lifetime of the $i$ th component. The intensity weighted average lifetime $\left(\tau_{\mathrm{Avg}}\right)$ was calculated using the following equation:

$$
\tau_{\mathrm{avg}}=\sum_{i} f_{i} \tau_{i} \quad \text { where } f_{i}=\frac{\alpha_{i} \tau_{i}}{\sum_{i} \alpha_{i} \tau_{i}}
$$

Radiative $\left(K_{\mathrm{r}}\right)$ and non-radiative $\left(K_{\mathrm{nr}}\right)$ rates were calculated using experimentally measured quantum yield and fluorescence lifetimes using the following equation:

$$
\phi_{\mathrm{f}}=\frac{K_{\mathrm{r}}}{K_{\mathrm{r}}+K_{\mathrm{nr}}} \text { and } \tau=\frac{1}{K_{\mathrm{r}}+K_{\mathrm{nr}}}
$$

\section{Preparation of lipid vesicles}

Three different lipid unilamellar vesicles were prepared using DPPC (1,2-dihexadecanoyl-sn-glycero-3-phosphocholine), POPC (1-hexadecanoyl-2-(9Z-octadecenoyl)-sn-glycero-3-phosphocholine) and POPC + cholesterol. Appropriate amounts of each lipid and the BODIPY homodimer were dissolved in chloroform (the lipid: dye ratio was $800: 1$ ) in glass bottles. The solvent was evaporated under oxygen free nitrogen stream and left overnight to remove any traces of organic solvents. Next, PBS (phosphate buffer saline) was added followed by strong sonication at about $40{ }^{\circ} \mathrm{C}$ to get giant multilamellar vesicles. Moreover, in order to obtain unilamellar vesicles, mutlilamellar vesicles were passed through $100 \mu \mathrm{m}$ and $0.02 \mu \mathrm{m}$ membrane syringe filters. As obtained lipid vesicles were used for fluorescence lifetime measurements.

\section{Fluorescence microscopy and FLIM}

The SKOV3 ovarian carcinoma cell line obtained from American Type Culture Collection (ATCC), Manassas, VA (USA), was grown to $70 \%$ confluence in RPMI supplemented with $10 \%$ FBS and $1 \%$ Pen-Strep. Cells were trypsinized using $0.25 \%$ Trypsin EDTA and seeded on $20 \mathrm{~mm}$ round glass-bottom petri dishes. After 24 hours, cells were stained with $500 \mathrm{nM}$ of BODIPY for $20 \mathrm{~min}$ at $37{ }^{\circ} \mathrm{C}$ followed by FLIM imaging on an Olympus IX7 microscope. Laser excitation was provided by a pulsed laser diode (PDL-470) emitting a $470 \mathrm{~nm}$ light and driven by a PDL 828 "Sepia II" driver. This driver was operated at $80 \mathrm{MHz}$. Measurements were performed using a MicroTime 200 time-resolved, confocal microscope from PicoQuant. The excitation and emission light was focused using a $60 \times 1.2$ NA Olympus objective in an Olympus IX71 microscope, and the emission light was filtered using a $488 \mathrm{~nm}$ long wave pass filter before passing through a $50 \mu \mathrm{m}$ pinhole. Detection was made using a hybrid photomultiplier assembly. The resolution of the time correlated single photon counting (TCSPC) module was set to 4 ps per bin in order to facilitate the detection at the highest possible resolution. All data analyses were performed using the SymPhoTime software, version 5.3.2. All experimental equipment and the SymPhoTime software were provided by PicoQuant, $\mathrm{GmbH}$, as part of the MicroTime 200 system.

\section{Theory}

In typical molecular rotors, a non-radiative pathway responds strongly to viscosity in the immediate vicinity of the rotor. 
Twisting or rotation within the molecular structure is the prime reason behind viscosity dependent behavior. A decrease in the non-radiative rate with increasing viscosity results in a sharp increase in fluorescence quantum yield and the fluorescence lifetime. In those cases where the spectroscopic characteristics of the probe are viscosity dependent, a linear dependence of the quantum yield as a function of viscosity should be observed. This viscosity dependence of quantum yield can be expressed using Forster-Hoffmann theory: ${ }^{26}$

$$
\ln \left(\phi_{\mathrm{f}}\right)=C+x \ln (n)
$$

where $n$ is the viscosity and $x$ is the slope, which is 0.6 for a perfect rotor as predicted by the Forster-Hoffmann theory. However, the main problem associated with using quantum yield-viscosity correlations is the inability to differentiate between viscosity and other factors, such as local dye concentration, which might affect the steady state intensity, and thus the quantum yield. On the other hand, the fluorescence lifetime can be utilized for the viscosity dependence without the aforementioned concern. Notably, a Forster-Hoffmann equation is also applicable to the lifetime and viscosity as:

$$
\ln (\tau)=C^{\prime}+y \ln (n)
$$

This equation serves as a basis to visualize the intracellular viscosity. A calibration curve of viscosity versus lifetime can be constructed using media of different viscosities and subsequently applied to map the viscosity inside the cells.

\section{Results and discussion}

\section{Photo-physical characterization}

Our goal was to establish whether a simple BODIPY homodimer could act as a molecular rotor, with its fluorescence emission and the fluorescence lifetime being sensitive to the ambient viscosity. It appeared that changing the viscosity of the media (by using ethanol-glycerol mixtures ${ }^{25}$ ) did not significantly affect the shape and peak emission wavelength (Fig. 2A). A small shift of the emission maximum $(\sim 7 \mathrm{~nm})$ was observed when viscosity changed from that of ethanol to that of glycerol and this could be ascribed to slight changes in the media polarity. Additionally, increasing the viscosity of the media increased the emission intensity and fluorescence quantum yield, which is consistent with eqn (4). The value of $\Phi_{\mathrm{f}}$, as a function of viscosity in ethanol:glycerol mixtures, is shown in Fig. 2B. Linear dependence of viscosity with regard to $\Phi_{\mathrm{f}}$ of the BODIPY dimer was observed for viscosities above $20 \mathrm{cP}$.

Fluorescence intensity decays at increasing viscosity in ethanol:glycerol mixtures were measured (Fig. 3A) and the fluorescence lifetime changed distinctly as a function of viscosity (Fig. 3B). Similar to quantum yield, linear dependence was observed for media's viscosity above $20 \mathrm{cP}$. Specifically, the fluorescence lifetime in ethanol $(1.2 \mathrm{cP})$ was $340 \mathrm{ps}$, while in glycerol $(1457 \mathrm{cP})$ the fluorescence lifetime increased to $4.3 \mathrm{~ns}$. The fluorescence lifetimes at viscosities between 1.2 and $1457 \mathrm{cP}$ were all above 300 ps. This is important, since the lower limit for
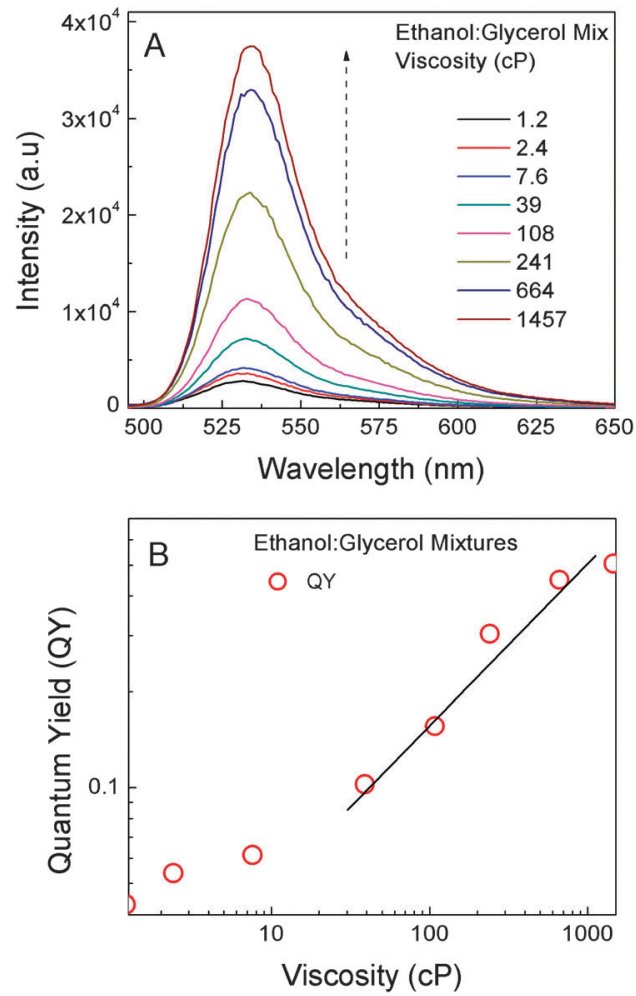

Fig. 2 (A) Emission spectra of the BODIPY dimer in different viscosity mixtures of ethanol:glycerol. (B) A log-log plot of quantum yield and viscosity.

lifetime resolution of our TCSPC (most of time-resolved systems available today) is significantly below 300 ps. Considering eqn (5), it was expected that the plot should have produced a linear dependence between the lifetime and log-viscosity. Indeed, this was confirmed at viscosities above $20 \mathrm{cP}$ giving a slope of 0.46 . This value compared well with the value of $2 / 3$ predicted by Forster and Hoffmann. ${ }^{26}$ Typical slope values range from 0.2 to 1.4 for different rotors. ${ }^{27}$ We found that below $20 \mathrm{cP}$, the fluorescence lifetime and quantum yield were minimally variant. Arguably, the viscosity dependent rotational resistance for BODIPY units in the dimer became insignificant at lower viscosities.

The rate constants, $K_{\mathrm{r}}$ and $K_{\mathrm{nr}}$, were calculated from the experimentally measured quantum yields and the fluorescence lifetime, using eqn (3). In ethanol, quantum yield was 0.02 and it increased significantly as the viscosity of the solution increased, as expected based on eqn (4). Moreover, the value of $K_{\mathrm{r}}$ remained steady as a function of the viscosity; however, the value of $K_{\mathrm{nr}}$ decreased sharply with increasing viscosity up to $375 \mathrm{cP}$ (Fig. 4A). This suggested that an increase in quantum yield with increasing viscosity was due to the suppression of non-radiative processes. Although the exact orientation of phenyl rings in relation to the plane of the BODIPY core remains to be clarified, it is arguable that the viscous environment prevented access to the non-emissive state by restricting the rotation of the BODIPY units around the diyne moiety. Efforts are underway to determine the absorption and emission 

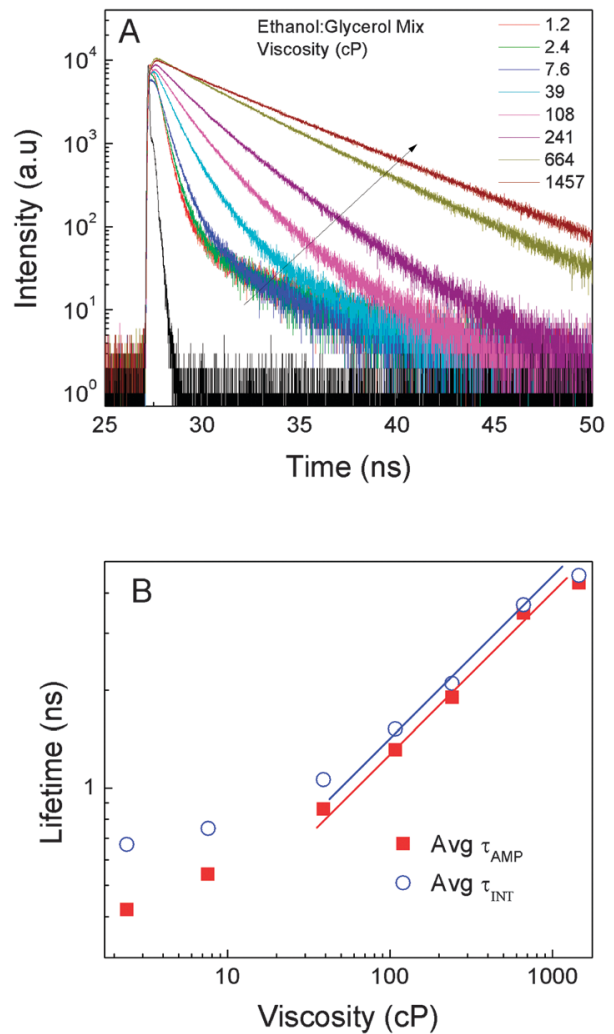

Fig. 3 (A) Fluorescence intensity decays of the BODIPY dimer in different viscosity mixtures of ethanol:glycerol. (B) Average fluorescence lifetime as a function of viscosity.

transition moments of the BODIPY dimer, which will further enlighten the exact mechanism of this molecular rotor. The complete viscosity dependence was further confirmed by the linear dependence of log quantum yield versus log lifetime (Fig. 4B).

Prior to the cellular studies, we envisioned that investigations using simple lipid vesicles would be advantageous for further understanding of the cellular observations. Thus, encapsulation of the BODIPY dimer into vesicles, which were made from different lipid components and physical states, could give an idea about the possible position of the dye molecules in lipid bilayers and their surrounding microviscosity from fluorescence lifetime measurements. Thus, the lifetime data of the BODIPY dimer in DPPC, POPC and POPC + cholesterol were evaluated (Fig. 5A). It appeared that average lifetimes did not vary significantly, except that POPC + cholesterol was found to be longer owing to a comparatively rigid membrane structure (Fig. 5B). Moreover, examining the lifetime distributions, DPPC indicated the presence of a more ordered lipid structure (as evident from a narrow lifetime distribution) whereby molecules were oriented in a certain (well-ordered) way compared to other two lipid vesicles. POPC showed a wider lifetime distribution attributed to different micro-viscosities experienced by the BODIPY dimer in vesicles due to less ordered lipid molecules in those vesicles.

We envisioned that using the fluorescence lifetime versus viscosity calibration plots obtained in ethanol:glycerol mixtures (Fig. 2-4) would allow us to determine the viscosity distribution
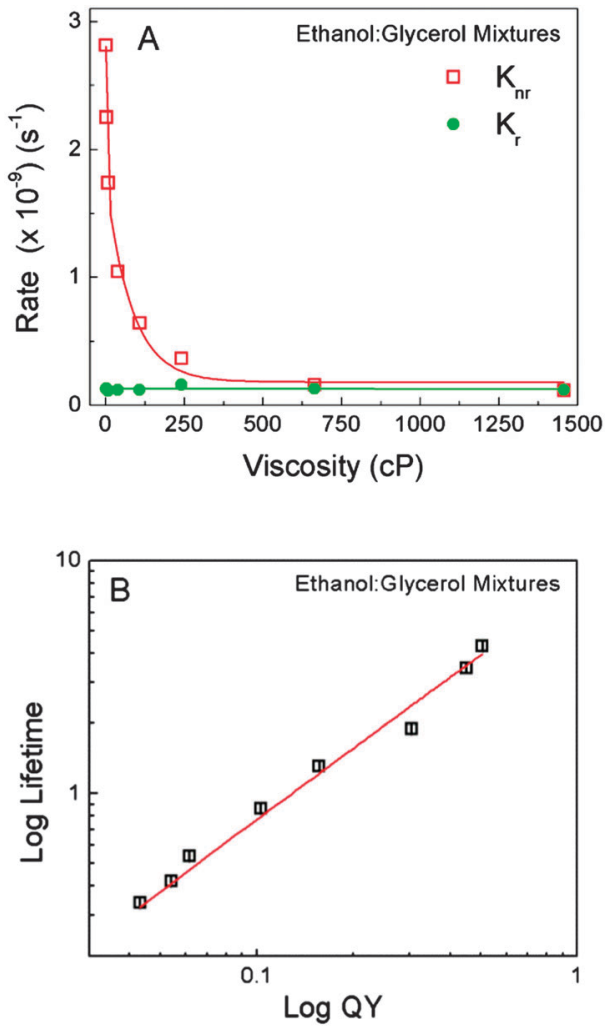

Fig. 4 (A) Radiative and non-radiative rates of the BODIPY dimer in ethanol:glycerol mixtures as a function of viscosity. (B) A log-log plot of the average fluorescence lifetime and quantum yield of the BODIPY dimer obtained from different ethanol:glycerol mixtures.

in cells via FLIM. Even though the fluorescence lifetime of a fluorophore could be influenced by several environmental parameters apart from viscosity, such as refractive index, polarity, $\mathrm{pH}$, or chemical and physical quenching processes, it was shown that BODIPY dyes were insensitive to changes in such environmental parameters and can be unambiguously used to probe the intracellular viscosity. ${ }^{18,28}$

\section{Fluorescence microscopy and FLIM}

The BODIPY dimer was readily taken up by the SKOV3 cells. A bright punctate distribution of the dye was observed throughout the cells. Moreover, very low intensity regions in cytosols were also present. We expected the BODIPY dimer to target the hydrophobic membrane regions owing to its hydrophobic nature as seen from lipid vesicle experiments. Punctate distribution appeared to be due to the accumulation of the dye in certain cell organelle membranes (Fig. 6A). This distribution pattern was found to be similar to the one observed by Levitt et al. ${ }^{18}$ the difference being less intense cytosolic fluorescence in our case. The lifetime distribution of the dye was examined after 20 min of the incubation time. Furthermore, with longer dye incubation time $(1 \mathrm{~h})$, no significant differences in distribution of the BODIPY dimer inside cells were observed. It is possible that the dye uptake mechanism might be a passive diffusion since the endocytotic uptake is energy dependent, yet the exact dye uptake mechanism remains to be clarified. 
A

\begin{tabular}{cccc}
\hline Lipid Vesicles & $\tau_{\mathrm{AMP}} / \mathrm{ns}$ & $\tau_{\mathrm{INT}} / \mathrm{ns}$ & Viscosity $/ \mathrm{cP}$ \\
\hline DPPC & 1.30 & 1.93 & 200 \\
POPC & 1.54 & 2.07 & 220 \\
POPC+cholesterol & 1.62 & 2.20 & 260
\end{tabular}

B
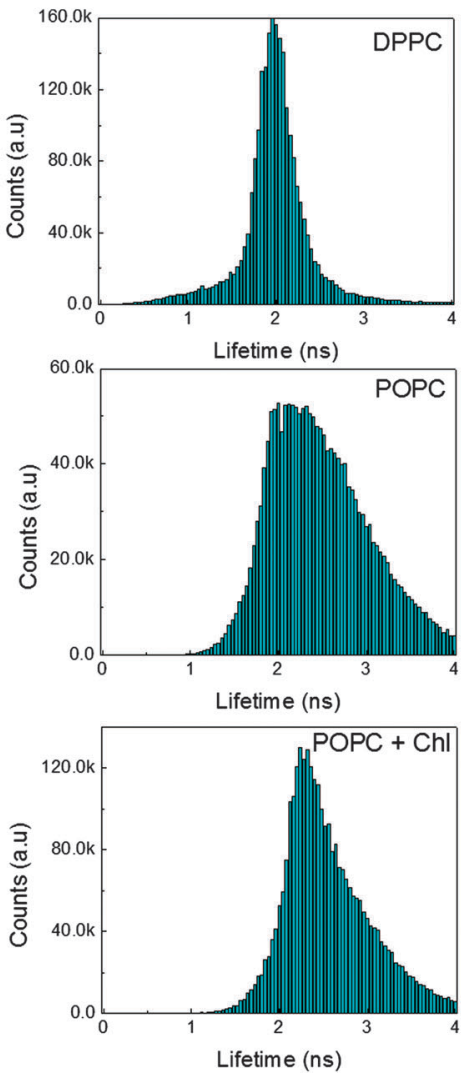

Fig. 5 (A) The table shows the average fluorescence lifetimes of the BODIPY dimer obtained in different lipid vesicles and corresponding viscosities calculated. (B) Lifetime distribution of the BODIPY dimer from lipid vesicles.

Following the calibration of fluorescence lifetimes of the BODIPY dimer as a function of viscosity, we performed FLIM analysis to generate a spatial map of viscosity in SKOV-3 cells incubated with the BODIPY dimer (Fig. 6B). Similar to the higher viscosity mixtures (ethanol:glycerol), the fluorescence decay in each pixel could be fitted with a bi-exponential distribution model. Earlier, we used such a model to study different conformational properties of adrenergic receptors. ${ }^{29}$ We obtained a histogram of fluorescence lifetimes across the whole image by graphing the lifetime distribution extracted from the image (Fig. 6C). The histogram showed the bi-modal distribution of the dye. By using a Gaussian fit, individual contributions to the histogram were 2.2 ns and 2.6 ns. Such lifetime distributions might indicate two distinctly different dye populations associated with different properties of the environments. A major part (ca. 90\%) was contributed by $2.2 \mathrm{~ns}$ with a narrow FWHM (Full Width Half Maximum), which was associated with the bright punctate distribution and appears to be located in the vesicular structures. The wider distribution of
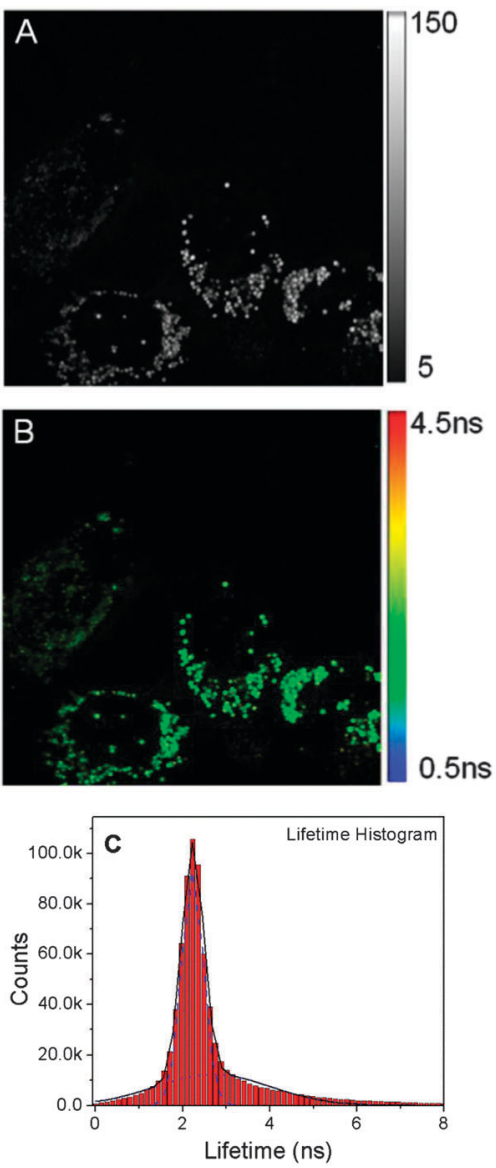

Fig. 6 (A) A confocal intensity image $(80 \times 80 \mu \mathrm{m})$ of SKOV3 cells treated with the BODIPY dimer. (B) A FLIM image of SKOV3 cells treated with the BODIPY dimer. (C) Lifetime distribution of SKOV3 cells treated with the BODIPY dimer obtained in (B).

the lifetime (2.6 ns peak) is due to the random distribution of the BODIPY dimer inside the cytoplasm and other cellular organelles (distribution of viscosities). The measured fluorescence lifetime inside cells lied within the linear range of the viscosity calibration plot. According to the calibration curve, the 2.2 ns lifetime appeared to correspond to the viscosity of $c a .260 \mathrm{cP}$.

The initial observation of the dye distribution indicated that these organelles could be either mitochondria or lysosomes. Thus, we decided to carry out the co-localization experiment using respective fluorescent markers and the BODIPY homodimer (Fig. 7): the two individual channels for the BODIPY
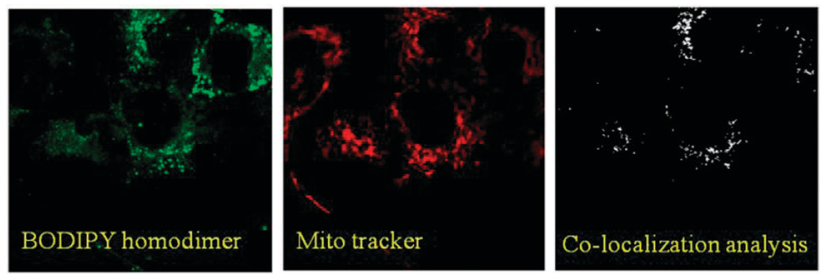

Fig. 7 Left panel: SKOV-3 cells treated with the BODIPY dimer; middle panel: SKOV-3 cells treated with Mitotracker; and right panel: co-localization analysis of both dyes using ImageJ. 

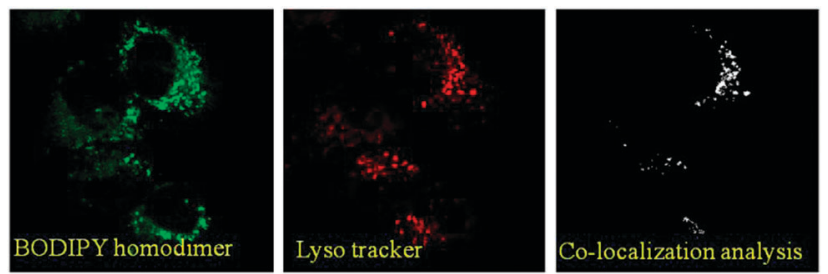

Fig. 8 Left panel: SKOV-3 cells treated with the BODIPY dimer; middle panel: SKOV-3 cells treated with Lysotracker; and right panel: co-localization analysis of both dyes using ImageJ.

dimer and Mitotracker along with co-localization analysis showed a partial overlap among them. Similar results were obtained using Lysotracker and the BODIPY dimer (Fig. 8). These observations suggested that the BODIPY rotor also accumulated in organelles other than mitochondria and lysosomes and thus exhibited nonspecific cell targeting.

\section{Conclusions}

In conclusion, we have reported on a novel, easily accessible homodimeric BODIPY as a steady state and time resolved viscosity sensitive molecular rotor, which allowed mapping of the viscosity by nonspecifically staining the intracellular organelle. Arguably, structural modifications of the rotor (for example, by incorporating long-alkyl chains onto the BODIPY scaffold) might allow for specific targeting of the cell organelle as well. Considering that the local microviscosity of the plasma membrane/cell organelle is altered in response to external stimuli (oxidants, pore forming agents and signaling events), these molecular viscometers could be suitable for diagnostic applications. These studies are underway in our laboratories.

\section{Acknowledgements}

This work was supported by the NIH grant RO1EB12003 (ZG), the NSF grant CBET-1264608 (IG), the NIH grant R15AG038977 (SVD) and the INFOR grant from Texas Christian University.

\section{Notes and References}

1 V. T. Turitto, Prog. Hemostasis Thromb., 1982, 6, 139-177.

2 Y. N. Andrade, J. Fernandes, E. Vazquez, J. M. FernandezFernandez, M. Arniges, T. M. Sanchez, M. Villalon and M. A. Valverde, J. Cell Biol., 2005, 168, 869-874.

3 M. K. Kuimova, Phys. Chem. Chem. Phys., 2012, 14, 12671-12686.

4 M. A. Haidekker and E. A. Theodorakis, Org. Biomol. Chem., 2007, 5, 1669-1678.

5 M. A. Haidekker and E. A. Theodorakis, J. Biol. Eng., 2010, 4, 11, http://www.jbioleng.org/content/4/1/11.
6 J. Fan, M. Hu, P. Zhan and X. Peng, Chem. Soc. Rev., 2013, 42, 29-43.

7 M. Kumar, N. Kumar, V. Bhalla, H. Singh, P. R. Sharma and T. Kaur, Org. Lett., 2011, 13, 1422-1425.

8 X. Cao, W. Lin, Q. Yu and J. Wang, Org. Lett., 2011, 13, 6098-6101.

9 H. Yu, Y. Xiao, H. Guo and X. Qian, Chem. - Eur. J., 2011, 17, 3179-3191.

10 G. Ulrich, R. Ziessel and A. Harriman, Angew. Chem., Int. Ed., 2008, 47, 1184-1201.

11 R. Ziessel, G. Ulrich and A. Harriman, New J. Chem., 2007, 31, 496-501.

12 N. Boens, V. Leen and W. Dehaen, Chem. Soc. Rev., 2012, 41, 1130-1172.

13 A. Loudet and K. Burgess, Chem. Rev., 2007, 107, 4891-4932.

14 A. Kamkaew, S. H. Lim, H. B. Lee, L. V. Kiew, L. Y. Chung and K. Burgess, Chem. Soc. Rev., 2013, 42, 77-88.

15 A. C. Benniston and G. Copley, Phys. Chem. Chem. Phys., 2009, 11, 4124-4131.

16 J. A. Levitt, P. Chung, M. K. Kuimova, G. Yahioglu, Y. Wang, J. Qu and K. Suhling, ChemPhysChem, 2011, 12, 662-672.

17 I. López-Duarte, T. T. Vu, M. A. Izquierdo, J. A. Bull and M. K. Kuimova, Chem. Commun., 2014, 50, 5282-5284.

18 J. A. Levitt, M. K. Kuimova, G. Yahioglu, P. Chung, K. Suhling and D. Phillips, J. Phys. Chem. C, 2009, 113, 11634-11642.

19 Y. Wu, M. Štefl, A. Olzyńska, M. Hof, G. Yahioglu, P. Yip, D. R. Casey, O. Ces, J. Humpolíčková and M. K. Kuimova, Phys. Chem. Chem. Phys., 2013, 15, 14986-14993.

20 H. Zhu, J. Fan, M. Li, J. Cao, J. Wang and X. Peng, Chem. Eur. J., 2014, 20, 4691-4696.

21 Z. Yang, Y. He, J. Lee, N. Park, M. Suh, W. Chae, J. Cao, X. Peng, H. Jung and C. Kang, J. Am. Chem. Soc., 2013, 135, 9181-9185.

22 M. K. Kuimova, S. W. Botchway, A. W. Parker, M. Balaz, H. A. Collins, H. L. Anderson, K. Suhling and P. R. Ogilby, Nat. Chem., 2009, 1, 69-73.

23 J. D. Kimball, S. Raut, L. P. Jameson, N. W. Smith, Z. Gryczynski and S. V. Dzyuba, RSC Adv., 2014, under review.

24 A. F. López, O. P. Ruiz and A. I. López, Chem. Phys. Lett., 1988, 148, 253-258.

25 L. P. Jameson, J. D. Kimball, Z. Gryczynski, M. Balaz and S. V. Dzyuba, RSC Adv., 2013, 3, 18300-18304.

26 T. Forster and G. Hoffmann, Z. Phys. Chem., 1971, 63-76.

27 M. Kaschke, J. Kleinschmidt and B. Wilhelmi, Laser Chem., 1985, 5, 119-132.

28 K. Suhling, P. M. French and D. Phillips, Photochem. Photobiol. Sci., 2005, 4, 13-22.

29 P. Ghanouni, Z. Gryczynski, J. Steenhuis, T. Lee, D. Farrens, J. Lakowicz and B. Kobilka, J. Biol. Chem., 2001, 276, 24433-24436. 